\title{
Categoricity Spectra for Rigid Structures
}

\author{
Ekaterina Fokina, Andrey Frolov, and Iskander Kalimullin
}

\begin{abstract}
For a computable structure $\mathcal{M}$, the categoricity spectrum is the set of all Turing degrees capable of computing isomorphisms among arbitrary computable copies of $\mathcal{M}$. If the spectrum has a least degree, this degree is called the degree of categoricity of $\mathcal{M}$. In this paper we investigate spectra of categoricity for computable rigid structures. In particular, we give examples of rigid structures without degrees of categoricity.
\end{abstract}

\section{Introduction}

We study algorithmic properties of isomorphisms between a computable structure $\mathcal{M}$ and its countable copies. A structure $\mathcal{A}$ is computable if $|\mathcal{A}|$ is a computable subset of $\omega$ and all basic predicates and functions are uniformly computable, or equivalently, the atomic diagram $\mathrm{D}(\mathcal{A})$, thought of as a subset of $\omega$, is computable.

We will make use of the following fact. Let $\sigma$ be a computable signature, and let $\sigma=\bigcup_{i} \sigma_{i}$ for a computable sequence of finite signatures $\sigma_{0} \subseteq \sigma_{1} \subseteq \cdots$. Let $\mathcal{M}$ be a countable structure in the signature $\sigma$. Then $\mathcal{M}$ is computable if and only if there exists a computable sequence $\left(\mathcal{M}_{i}\right)_{i \in \omega}$ of finite structures such that

1. $\mathcal{M}=\bigcup_{i} \mathcal{M}_{i}$,

2. $\mathcal{M}_{i} \subseteq \mathcal{M}_{i+1}$, for all $i$, and

3. each $\mathcal{M}_{i}$ is a $\sigma_{i}$-structure with domain $\left\{0, \ldots, t_{i}\right\}$, where the function sending $i$ to $t_{i}$ is computable.

In other words, a computable structure is a structure that may be effectively constructed step by step, where at each step we define an ever larger finite piece of the structure.

In this paper we are interested in complexity of isomorphisms between computable presentations of a countable structure. The main notion in this area of in-

Received February 14, 2013; accepted August 26, 2013

First published online October 30, 2015

2010 Mathematics Subject Classification: Primary 03C57, 03D45

Keywords: computable structure, rigid structure, computably categorical, categoricity spectrum, degree of categoricity

(C) 2016 by University of Notre Dame 10.1215/00294527-3322017 


\title{
Categoricity Spectra for Rigid Structures
}

\author{
Ekaterina Fokina, Andrey Frolov, and Iskander Kalimullin
}

\begin{abstract}
For a computable structure $\mathcal{M}$, the categoricity spectrum is the set of all Turing degrees capable of computing isomorphisms among arbitrary computable copies of $\mathcal{M}$. If the spectrum has a least degree, this degree is called the degree of categoricity of $\mathcal{M}$. In this paper we investigate spectra of categoricity for computable rigid structures. In particular, we give examples of rigid structures without degrees of categoricity.
\end{abstract}

\section{Introduction}

We study algorithmic properties of isomorphisms between a computable structure $\mathcal{M}$ and its countable copies. A structure $\mathcal{A}$ is computable if $|\mathcal{A}|$ is a computable subset of $\omega$ and all basic predicates and functions are uniformly computable, or equivalently, the atomic diagram $\mathrm{D}(\mathcal{A})$, thought of as a subset of $\omega$, is computable.

We will make use of the following fact. Let $\sigma$ be a computable signature, and let $\sigma=\bigcup_{i} \sigma_{i}$ for a computable sequence of finite signatures $\sigma_{0} \subseteq \sigma_{1} \subseteq \cdots$. Let $\mathcal{M}$ be a countable structure in the signature $\sigma$. Then $\mathcal{M}$ is computable if and only if there exists a computable sequence $\left(\mathcal{M}_{i}\right)_{i \in \omega}$ of finite structures such that

1. $\mathcal{M}=\bigcup_{i} \mathcal{M}_{i}$,

2. $\mathcal{M}_{i} \subseteq \mathcal{M}_{i+1}$, for all $i$, and

3. each $\mathcal{M}_{i}$ is a $\sigma_{i}$-structure with domain $\left\{0, \ldots, t_{i}\right\}$, where the function sending $i$ to $t_{i}$ is computable.

In other words, a computable structure is a structure that may be effectively constructed step by step, where at each step we define an ever larger finite piece of the structure.

In this paper we are interested in complexity of isomorphisms between computable presentations of a countable structure. The main notion in this area of in-

Received February 14, 2013; accepted August 26, 2013

First published online October 30, 2015

2010 Mathematics Subject Classification: Primary 03C57, 03D45

Keywords: computable structure, rigid structure, computably categorical, categoricity spectrum, degree of categoricity

(C) 2016 by University of Notre Dame 10.1215/00294527-3322017 


\title{
Categoricity Spectra for Rigid Structures
}

\author{
Ekaterina Fokina, Andrey Frolov, and Iskander Kalimullin
}

\begin{abstract}
For a computable structure $\mathcal{M}$, the categoricity spectrum is the set of all Turing degrees capable of computing isomorphisms among arbitrary computable copies of $\mathcal{M}$. If the spectrum has a least degree, this degree is called the degree of categoricity of $\mathcal{M}$. In this paper we investigate spectra of categoricity for computable rigid structures. In particular, we give examples of rigid structures without degrees of categoricity.
\end{abstract}

\section{Introduction}

We study algorithmic properties of isomorphisms between a computable structure $\mathcal{M}$ and its countable copies. A structure $\mathcal{A}$ is computable if $|\mathcal{A}|$ is a computable subset of $\omega$ and all basic predicates and functions are uniformly computable, or equivalently, the atomic diagram $\mathrm{D}(\mathcal{A})$, thought of as a subset of $\omega$, is computable.

We will make use of the following fact. Let $\sigma$ be a computable signature, and let $\sigma=\bigcup_{i} \sigma_{i}$ for a computable sequence of finite signatures $\sigma_{0} \subseteq \sigma_{1} \subseteq \cdots$. Let $\mathcal{M}$ be a countable structure in the signature $\sigma$. Then $\mathcal{M}$ is computable if and only if there exists a computable sequence $\left(\mathcal{M}_{i}\right)_{i \in \omega}$ of finite structures such that

1. $\mathcal{M}=\bigcup_{i} \mathcal{M}_{i}$,

2. $\mathcal{M}_{i} \subseteq \mathcal{M}_{i+1}$, for all $i$, and

3. each $\mathcal{M}_{i}$ is a $\sigma_{i}$-structure with domain $\left\{0, \ldots, t_{i}\right\}$, where the function sending $i$ to $t_{i}$ is computable.

In other words, a computable structure is a structure that may be effectively constructed step by step, where at each step we define an ever larger finite piece of the structure.

In this paper we are interested in complexity of isomorphisms between computable presentations of a countable structure. The main notion in this area of in-

Received February 14, 2013; accepted August 26, 2013

First published online October 30, 2015

2010 Mathematics Subject Classification: Primary 03C57, 03D45

Keywords: computable structure, rigid structure, computably categorical, categoricity spectrum, degree of categoricity

(C) 2016 by University of Notre Dame 10.1215/00294527-3322017 


\title{
Categoricity Spectra for Rigid Structures
}

\author{
Ekaterina Fokina, Andrey Frolov, and Iskander Kalimullin
}

\begin{abstract}
For a computable structure $\mathcal{M}$, the categoricity spectrum is the set of all Turing degrees capable of computing isomorphisms among arbitrary computable copies of $\mathcal{M}$. If the spectrum has a least degree, this degree is called the degree of categoricity of $\mathcal{M}$. In this paper we investigate spectra of categoricity for computable rigid structures. In particular, we give examples of rigid structures without degrees of categoricity.
\end{abstract}

\section{Introduction}

We study algorithmic properties of isomorphisms between a computable structure $\mathcal{M}$ and its countable copies. A structure $\mathcal{A}$ is computable if $|\mathcal{A}|$ is a computable subset of $\omega$ and all basic predicates and functions are uniformly computable, or equivalently, the atomic diagram $\mathrm{D}(\mathcal{A})$, thought of as a subset of $\omega$, is computable.

We will make use of the following fact. Let $\sigma$ be a computable signature, and let $\sigma=\bigcup_{i} \sigma_{i}$ for a computable sequence of finite signatures $\sigma_{0} \subseteq \sigma_{1} \subseteq \cdots$. Let $\mathcal{M}$ be a countable structure in the signature $\sigma$. Then $\mathcal{M}$ is computable if and only if there exists a computable sequence $\left(\mathcal{M}_{i}\right)_{i \in \omega}$ of finite structures such that

1. $\mathcal{M}=\bigcup_{i} \mathcal{M}_{i}$,

2. $\mathcal{M}_{i} \subseteq \mathcal{M}_{i+1}$, for all $i$, and

3. each $\mathcal{M}_{i}$ is a $\sigma_{i}$-structure with domain $\left\{0, \ldots, t_{i}\right\}$, where the function sending $i$ to $t_{i}$ is computable.

In other words, a computable structure is a structure that may be effectively constructed step by step, where at each step we define an ever larger finite piece of the structure.

In this paper we are interested in complexity of isomorphisms between computable presentations of a countable structure. The main notion in this area of in-

Received February 14, 2013; accepted August 26, 2013

First published online October 30, 2015

2010 Mathematics Subject Classification: Primary 03C57, 03D45

Keywords: computable structure, rigid structure, computably categorical, categoricity spectrum, degree of categoricity

(C) 2016 by University of Notre Dame 10.1215/00294527-3322017 


\title{
Categoricity Spectra for Rigid Structures
}

\author{
Ekaterina Fokina, Andrey Frolov, and Iskander Kalimullin
}

\begin{abstract}
For a computable structure $\mathcal{M}$, the categoricity spectrum is the set of all Turing degrees capable of computing isomorphisms among arbitrary computable copies of $\mathcal{M}$. If the spectrum has a least degree, this degree is called the degree of categoricity of $\mathcal{M}$. In this paper we investigate spectra of categoricity for computable rigid structures. In particular, we give examples of rigid structures without degrees of categoricity.
\end{abstract}

\section{Introduction}

We study algorithmic properties of isomorphisms between a computable structure $\mathcal{M}$ and its countable copies. A structure $\mathcal{A}$ is computable if $|\mathcal{A}|$ is a computable subset of $\omega$ and all basic predicates and functions are uniformly computable, or equivalently, the atomic diagram $\mathrm{D}(\mathcal{A})$, thought of as a subset of $\omega$, is computable.

We will make use of the following fact. Let $\sigma$ be a computable signature, and let $\sigma=\bigcup_{i} \sigma_{i}$ for a computable sequence of finite signatures $\sigma_{0} \subseteq \sigma_{1} \subseteq \cdots$. Let $\mathcal{M}$ be a countable structure in the signature $\sigma$. Then $\mathcal{M}$ is computable if and only if there exists a computable sequence $\left(\mathcal{M}_{i}\right)_{i \in \omega}$ of finite structures such that

1. $\mathcal{M}=\bigcup_{i} \mathcal{M}_{i}$,

2. $\mathcal{M}_{i} \subseteq \mathcal{M}_{i+1}$, for all $i$, and

3. each $\mathcal{M}_{i}$ is a $\sigma_{i}$-structure with domain $\left\{0, \ldots, t_{i}\right\}$, where the function sending $i$ to $t_{i}$ is computable.

In other words, a computable structure is a structure that may be effectively constructed step by step, where at each step we define an ever larger finite piece of the structure.

In this paper we are interested in complexity of isomorphisms between computable presentations of a countable structure. The main notion in this area of in-

Received February 14, 2013; accepted August 26, 2013

First published online October 30, 2015

2010 Mathematics Subject Classification: Primary 03C57, 03D45

Keywords: computable structure, rigid structure, computably categorical, categoricity spectrum, degree of categoricity

(C) 2016 by University of Notre Dame 10.1215/00294527-3322017 


\title{
Categoricity Spectra for Rigid Structures
}

\author{
Ekaterina Fokina, Andrey Frolov, and Iskander Kalimullin
}

\begin{abstract}
For a computable structure $\mathcal{M}$, the categoricity spectrum is the set of all Turing degrees capable of computing isomorphisms among arbitrary computable copies of $\mathcal{M}$. If the spectrum has a least degree, this degree is called the degree of categoricity of $\mathcal{M}$. In this paper we investigate spectra of categoricity for computable rigid structures. In particular, we give examples of rigid structures without degrees of categoricity.
\end{abstract}

\section{Introduction}

We study algorithmic properties of isomorphisms between a computable structure $\mathcal{M}$ and its countable copies. A structure $\mathcal{A}$ is computable if $|\mathcal{A}|$ is a computable subset of $\omega$ and all basic predicates and functions are uniformly computable, or equivalently, the atomic diagram $\mathrm{D}(\mathcal{A})$, thought of as a subset of $\omega$, is computable.

We will make use of the following fact. Let $\sigma$ be a computable signature, and let $\sigma=\bigcup_{i} \sigma_{i}$ for a computable sequence of finite signatures $\sigma_{0} \subseteq \sigma_{1} \subseteq \cdots$. Let $\mathcal{M}$ be a countable structure in the signature $\sigma$. Then $\mathcal{M}$ is computable if and only if there exists a computable sequence $\left(\mathcal{M}_{i}\right)_{i \in \omega}$ of finite structures such that

1. $\mathcal{M}=\bigcup_{i} \mathcal{M}_{i}$,

2. $\mathcal{M}_{i} \subseteq \mathcal{M}_{i+1}$, for all $i$, and

3. each $\mathcal{M}_{i}$ is a $\sigma_{i}$-structure with domain $\left\{0, \ldots, t_{i}\right\}$, where the function sending $i$ to $t_{i}$ is computable.

In other words, a computable structure is a structure that may be effectively constructed step by step, where at each step we define an ever larger finite piece of the structure.

In this paper we are interested in complexity of isomorphisms between computable presentations of a countable structure. The main notion in this area of in-

Received February 14, 2013; accepted August 26, 2013

First published online October 30, 2015

2010 Mathematics Subject Classification: Primary 03C57, 03D45

Keywords: computable structure, rigid structure, computably categorical, categoricity spectrum, degree of categoricity

(C) 2016 by University of Notre Dame 10.1215/00294527-3322017 


\title{
Categoricity Spectra for Rigid Structures
}

\author{
Ekaterina Fokina, Andrey Frolov, and Iskander Kalimullin
}

\begin{abstract}
For a computable structure $\mathcal{M}$, the categoricity spectrum is the set of all Turing degrees capable of computing isomorphisms among arbitrary computable copies of $\mathcal{M}$. If the spectrum has a least degree, this degree is called the degree of categoricity of $\mathcal{M}$. In this paper we investigate spectra of categoricity for computable rigid structures. In particular, we give examples of rigid structures without degrees of categoricity.
\end{abstract}

\section{Introduction}

We study algorithmic properties of isomorphisms between a computable structure $\mathcal{M}$ and its countable copies. A structure $\mathcal{A}$ is computable if $|\mathcal{A}|$ is a computable subset of $\omega$ and all basic predicates and functions are uniformly computable, or equivalently, the atomic diagram $\mathrm{D}(\mathcal{A})$, thought of as a subset of $\omega$, is computable.

We will make use of the following fact. Let $\sigma$ be a computable signature, and let $\sigma=\bigcup_{i} \sigma_{i}$ for a computable sequence of finite signatures $\sigma_{0} \subseteq \sigma_{1} \subseteq \cdots$. Let $\mathcal{M}$ be a countable structure in the signature $\sigma$. Then $\mathcal{M}$ is computable if and only if there exists a computable sequence $\left(\mathcal{M}_{i}\right)_{i \in \omega}$ of finite structures such that

1. $\mathcal{M}=\bigcup_{i} \mathcal{M}_{i}$,

2. $\mathcal{M}_{i} \subseteq \mathcal{M}_{i+1}$, for all $i$, and

3. each $\mathcal{M}_{i}$ is a $\sigma_{i}$-structure with domain $\left\{0, \ldots, t_{i}\right\}$, where the function sending $i$ to $t_{i}$ is computable.

In other words, a computable structure is a structure that may be effectively constructed step by step, where at each step we define an ever larger finite piece of the structure.

In this paper we are interested in complexity of isomorphisms between computable presentations of a countable structure. The main notion in this area of in-

Received February 14, 2013; accepted August 26, 2013

First published online October 30, 2015

2010 Mathematics Subject Classification: Primary 03C57, 03D45

Keywords: computable structure, rigid structure, computably categorical, categoricity spectrum, degree of categoricity

(C) 2016 by University of Notre Dame 10.1215/00294527-3322017 


\title{
Categoricity Spectra for Rigid Structures
}

\author{
Ekaterina Fokina, Andrey Frolov, and Iskander Kalimullin
}

\begin{abstract}
For a computable structure $\mathcal{M}$, the categoricity spectrum is the set of all Turing degrees capable of computing isomorphisms among arbitrary computable copies of $\mathcal{M}$. If the spectrum has a least degree, this degree is called the degree of categoricity of $\mathcal{M}$. In this paper we investigate spectra of categoricity for computable rigid structures. In particular, we give examples of rigid structures without degrees of categoricity.
\end{abstract}

\section{Introduction}

We study algorithmic properties of isomorphisms between a computable structure $\mathcal{M}$ and its countable copies. A structure $\mathcal{A}$ is computable if $|\mathcal{A}|$ is a computable subset of $\omega$ and all basic predicates and functions are uniformly computable, or equivalently, the atomic diagram $\mathrm{D}(\mathcal{A})$, thought of as a subset of $\omega$, is computable.

We will make use of the following fact. Let $\sigma$ be a computable signature, and let $\sigma=\bigcup_{i} \sigma_{i}$ for a computable sequence of finite signatures $\sigma_{0} \subseteq \sigma_{1} \subseteq \cdots$. Let $\mathcal{M}$ be a countable structure in the signature $\sigma$. Then $\mathcal{M}$ is computable if and only if there exists a computable sequence $\left(\mathcal{M}_{i}\right)_{i \in \omega}$ of finite structures such that

1. $\mathcal{M}=\bigcup_{i} \mathcal{M}_{i}$,

2. $\mathcal{M}_{i} \subseteq \mathcal{M}_{i+1}$, for all $i$, and

3. each $\mathcal{M}_{i}$ is a $\sigma_{i}$-structure with domain $\left\{0, \ldots, t_{i}\right\}$, where the function sending $i$ to $t_{i}$ is computable.

In other words, a computable structure is a structure that may be effectively constructed step by step, where at each step we define an ever larger finite piece of the structure.

In this paper we are interested in complexity of isomorphisms between computable presentations of a countable structure. The main notion in this area of in-

Received February 14, 2013; accepted August 26, 2013

First published online October 30, 2015

2010 Mathematics Subject Classification: Primary 03C57, 03D45

Keywords: computable structure, rigid structure, computably categorical, categoricity spectrum, degree of categoricity

(C) 2016 by University of Notre Dame 10.1215/00294527-3322017 


\title{
Categoricity Spectra for Rigid Structures
}

\author{
Ekaterina Fokina, Andrey Frolov, and Iskander Kalimullin
}

\begin{abstract}
For a computable structure $\mathcal{M}$, the categoricity spectrum is the set of all Turing degrees capable of computing isomorphisms among arbitrary computable copies of $\mathcal{M}$. If the spectrum has a least degree, this degree is called the degree of categoricity of $\mathcal{M}$. In this paper we investigate spectra of categoricity for computable rigid structures. In particular, we give examples of rigid structures without degrees of categoricity.
\end{abstract}

\section{Introduction}

We study algorithmic properties of isomorphisms between a computable structure $\mathcal{M}$ and its countable copies. A structure $\mathcal{A}$ is computable if $|\mathcal{A}|$ is a computable subset of $\omega$ and all basic predicates and functions are uniformly computable, or equivalently, the atomic diagram $\mathrm{D}(\mathcal{A})$, thought of as a subset of $\omega$, is computable.

We will make use of the following fact. Let $\sigma$ be a computable signature, and let $\sigma=\bigcup_{i} \sigma_{i}$ for a computable sequence of finite signatures $\sigma_{0} \subseteq \sigma_{1} \subseteq \cdots$. Let $\mathcal{M}$ be a countable structure in the signature $\sigma$. Then $\mathcal{M}$ is computable if and only if there exists a computable sequence $\left(\mathcal{M}_{i}\right)_{i \in \omega}$ of finite structures such that

1. $\mathcal{M}=\bigcup_{i} \mathcal{M}_{i}$,

2. $\mathcal{M}_{i} \subseteq \mathcal{M}_{i+1}$, for all $i$, and

3. each $\mathcal{M}_{i}$ is a $\sigma_{i}$-structure with domain $\left\{0, \ldots, t_{i}\right\}$, where the function sending $i$ to $t_{i}$ is computable.

In other words, a computable structure is a structure that may be effectively constructed step by step, where at each step we define an ever larger finite piece of the structure.

In this paper we are interested in complexity of isomorphisms between computable presentations of a countable structure. The main notion in this area of in-

Received February 14, 2013; accepted August 26, 2013

First published online October 30, 2015

2010 Mathematics Subject Classification: Primary 03C57, 03D45

Keywords: computable structure, rigid structure, computably categorical, categoricity spectrum, degree of categoricity

(C) 2016 by University of Notre Dame 10.1215/00294527-3322017 


\title{
Categoricity Spectra for Rigid Structures
}

\author{
Ekaterina Fokina, Andrey Frolov, and Iskander Kalimullin
}

\begin{abstract}
For a computable structure $\mathcal{M}$, the categoricity spectrum is the set of all Turing degrees capable of computing isomorphisms among arbitrary computable copies of $\mathcal{M}$. If the spectrum has a least degree, this degree is called the degree of categoricity of $\mathcal{M}$. In this paper we investigate spectra of categoricity for computable rigid structures. In particular, we give examples of rigid structures without degrees of categoricity.
\end{abstract}

\section{Introduction}

We study algorithmic properties of isomorphisms between a computable structure $\mathcal{M}$ and its countable copies. A structure $\mathcal{A}$ is computable if $|\mathcal{A}|$ is a computable subset of $\omega$ and all basic predicates and functions are uniformly computable, or equivalently, the atomic diagram $\mathrm{D}(\mathcal{A})$, thought of as a subset of $\omega$, is computable.

We will make use of the following fact. Let $\sigma$ be a computable signature, and let $\sigma=\bigcup_{i} \sigma_{i}$ for a computable sequence of finite signatures $\sigma_{0} \subseteq \sigma_{1} \subseteq \cdots$. Let $\mathcal{M}$ be a countable structure in the signature $\sigma$. Then $\mathcal{M}$ is computable if and only if there exists a computable sequence $\left(\mathcal{M}_{i}\right)_{i \in \omega}$ of finite structures such that

1. $\mathcal{M}=\bigcup_{i} \mathcal{M}_{i}$,

2. $\mathcal{M}_{i} \subseteq \mathcal{M}_{i+1}$, for all $i$, and

3. each $\mathcal{M}_{i}$ is a $\sigma_{i}$-structure with domain $\left\{0, \ldots, t_{i}\right\}$, where the function sending $i$ to $t_{i}$ is computable.

In other words, a computable structure is a structure that may be effectively constructed step by step, where at each step we define an ever larger finite piece of the structure.

In this paper we are interested in complexity of isomorphisms between computable presentations of a countable structure. The main notion in this area of in-

Received February 14, 2013; accepted August 26, 2013

First published online October 30, 2015

2010 Mathematics Subject Classification: Primary 03C57, 03D45

Keywords: computable structure, rigid structure, computably categorical, categoricity spectrum, degree of categoricity

(C) 2016 by University of Notre Dame 10.1215/00294527-3322017 


\title{
Categoricity Spectra for Rigid Structures
}

\author{
Ekaterina Fokina, Andrey Frolov, and Iskander Kalimullin
}

\begin{abstract}
For a computable structure $\mathcal{M}$, the categoricity spectrum is the set of all Turing degrees capable of computing isomorphisms among arbitrary computable copies of $\mathcal{M}$. If the spectrum has a least degree, this degree is called the degree of categoricity of $\mathcal{M}$. In this paper we investigate spectra of categoricity for computable rigid structures. In particular, we give examples of rigid structures without degrees of categoricity.
\end{abstract}

\section{Introduction}

We study algorithmic properties of isomorphisms between a computable structure $\mathcal{M}$ and its countable copies. A structure $\mathcal{A}$ is computable if $|\mathcal{A}|$ is a computable subset of $\omega$ and all basic predicates and functions are uniformly computable, or equivalently, the atomic diagram $\mathrm{D}(\mathcal{A})$, thought of as a subset of $\omega$, is computable.

We will make use of the following fact. Let $\sigma$ be a computable signature, and let $\sigma=\bigcup_{i} \sigma_{i}$ for a computable sequence of finite signatures $\sigma_{0} \subseteq \sigma_{1} \subseteq \cdots$. Let $\mathcal{M}$ be a countable structure in the signature $\sigma$. Then $\mathcal{M}$ is computable if and only if there exists a computable sequence $\left(\mathcal{M}_{i}\right)_{i \in \omega}$ of finite structures such that

1. $\mathcal{M}=\bigcup_{i} \mathcal{M}_{i}$,

2. $\mathcal{M}_{i} \subseteq \mathcal{M}_{i+1}$, for all $i$, and

3. each $\mathcal{M}_{i}$ is a $\sigma_{i}$-structure with domain $\left\{0, \ldots, t_{i}\right\}$, where the function sending $i$ to $t_{i}$ is computable.

In other words, a computable structure is a structure that may be effectively constructed step by step, where at each step we define an ever larger finite piece of the structure.

In this paper we are interested in complexity of isomorphisms between computable presentations of a countable structure. The main notion in this area of in-

Received February 14, 2013; accepted August 26, 2013

First published online October 30, 2015

2010 Mathematics Subject Classification: Primary 03C57, 03D45

Keywords: computable structure, rigid structure, computably categorical, categoricity spectrum, degree of categoricity

(C) 2016 by University of Notre Dame 10.1215/00294527-3322017 


\title{
Categoricity Spectra for Rigid Structures
}

\author{
Ekaterina Fokina, Andrey Frolov, and Iskander Kalimullin
}

\begin{abstract}
For a computable structure $\mathcal{M}$, the categoricity spectrum is the set of all Turing degrees capable of computing isomorphisms among arbitrary computable copies of $\mathcal{M}$. If the spectrum has a least degree, this degree is called the degree of categoricity of $\mathcal{M}$. In this paper we investigate spectra of categoricity for computable rigid structures. In particular, we give examples of rigid structures without degrees of categoricity.
\end{abstract}

\section{Introduction}

We study algorithmic properties of isomorphisms between a computable structure $\mathcal{M}$ and its countable copies. A structure $\mathcal{A}$ is computable if $|\mathcal{A}|$ is a computable subset of $\omega$ and all basic predicates and functions are uniformly computable, or equivalently, the atomic diagram $\mathrm{D}(\mathcal{A})$, thought of as a subset of $\omega$, is computable.

We will make use of the following fact. Let $\sigma$ be a computable signature, and let $\sigma=\bigcup_{i} \sigma_{i}$ for a computable sequence of finite signatures $\sigma_{0} \subseteq \sigma_{1} \subseteq \cdots$. Let $\mathcal{M}$ be a countable structure in the signature $\sigma$. Then $\mathcal{M}$ is computable if and only if there exists a computable sequence $\left(\mathcal{M}_{i}\right)_{i \in \omega}$ of finite structures such that

1. $\mathcal{M}=\bigcup_{i} \mathcal{M}_{i}$,

2. $\mathcal{M}_{i} \subseteq \mathcal{M}_{i+1}$, for all $i$, and

3. each $\mathcal{M}_{i}$ is a $\sigma_{i}$-structure with domain $\left\{0, \ldots, t_{i}\right\}$, where the function sending $i$ to $t_{i}$ is computable.

In other words, a computable structure is a structure that may be effectively constructed step by step, where at each step we define an ever larger finite piece of the structure.

In this paper we are interested in complexity of isomorphisms between computable presentations of a countable structure. The main notion in this area of in-

Received February 14, 2013; accepted August 26, 2013

First published online October 30, 2015

2010 Mathematics Subject Classification: Primary 03C57, 03D45

Keywords: computable structure, rigid structure, computably categorical, categoricity spectrum, degree of categoricity

(C) 2016 by University of Notre Dame 10.1215/00294527-3322017 


\title{
Categoricity Spectra for Rigid Structures
}

\author{
Ekaterina Fokina, Andrey Frolov, and Iskander Kalimullin
}

\begin{abstract}
For a computable structure $\mathcal{M}$, the categoricity spectrum is the set of all Turing degrees capable of computing isomorphisms among arbitrary computable copies of $\mathcal{M}$. If the spectrum has a least degree, this degree is called the degree of categoricity of $\mathcal{M}$. In this paper we investigate spectra of categoricity for computable rigid structures. In particular, we give examples of rigid structures without degrees of categoricity.
\end{abstract}

\section{Introduction}

We study algorithmic properties of isomorphisms between a computable structure $\mathcal{M}$ and its countable copies. A structure $\mathcal{A}$ is computable if $|\mathcal{A}|$ is a computable subset of $\omega$ and all basic predicates and functions are uniformly computable, or equivalently, the atomic diagram $\mathrm{D}(\mathcal{A})$, thought of as a subset of $\omega$, is computable.

We will make use of the following fact. Let $\sigma$ be a computable signature, and let $\sigma=\bigcup_{i} \sigma_{i}$ for a computable sequence of finite signatures $\sigma_{0} \subseteq \sigma_{1} \subseteq \cdots$. Let $\mathcal{M}$ be a countable structure in the signature $\sigma$. Then $\mathcal{M}$ is computable if and only if there exists a computable sequence $\left(\mathcal{M}_{i}\right)_{i \in \omega}$ of finite structures such that

1. $\mathcal{M}=\bigcup_{i} \mathcal{M}_{i}$,

2. $\mathcal{M}_{i} \subseteq \mathcal{M}_{i+1}$, for all $i$, and

3. each $\mathcal{M}_{i}$ is a $\sigma_{i}$-structure with domain $\left\{0, \ldots, t_{i}\right\}$, where the function sending $i$ to $t_{i}$ is computable.

In other words, a computable structure is a structure that may be effectively constructed step by step, where at each step we define an ever larger finite piece of the structure.

In this paper we are interested in complexity of isomorphisms between computable presentations of a countable structure. The main notion in this area of in-

Received February 14, 2013; accepted August 26, 2013

First published online October 30, 2015

2010 Mathematics Subject Classification: Primary 03C57, 03D45

Keywords: computable structure, rigid structure, computably categorical, categoricity spectrum, degree of categoricity

(C) 2016 by University of Notre Dame 10.1215/00294527-3322017 